\title{
Retrosigmoid Craniotomy for Resection of an Epidermoid Cyst of the Posterior Fossa
}

\author{
M. Yashar S. Kalani ${ }^{1}$ William T. Couldwell ${ }^{2}$
}

\begin{abstract}
Address for correspondence M. Yashar S. Kalani, MD, PhD, Department of Neurosurgery, University of Virginia, School of Medicine, PO Box 800212, Charlottesville, VA 22908, United States (e-mail: kalani@virginia.edu).
\end{abstract}

J Neurol Surg B 2018;79(suppl S5):S411-S412.

\begin{abstract}
Keywords

- retrosigmoid

- cerebellopontine angle

- epidermoid

This video illustrates the case of a 51-year-old woman who presented with sudden-onset headache, vertigo, and nausea. Imaging revealed an epidermoid cyst of the posterior fossa with mass effect upon the brainstem and displacement of the basilar artery. This lesion was approached using a left-sided keyhole retrosigmoid craniotomy with monitoring of the cranial nerves. This video illustrates the technique of internal debulking of the cyst contents with minimal manipulation of the cyst capsule, which is often densely adherent to the brainstem, cranial nerves, and vessels in the posterior fossa. Resection of the capsule is often associated with a higher rate of cranial nerve deficits. The tumor was removed completely, but the cyst capsule was left in place. The patient had House-Brackmann grade II facial paralysis postoperatively and complained of some diminished hearing in the left ear. Epidermoid cysts are benign tumors, but the patient may experience much morbidity from their overly aggressive resection, especially when the capsule is densely adhering to critical structures. An alternate strategy is to decompress the contents of the epidermoid cyst, thereby decompressing the brainstem and converting this disease process into a chronic disease that may require reoperation in the long term. Given the tight confines of the posterior fossa, aggressive internal decompression of tumors and mobilization from the brainstem and adjacent nerves are key to avoiding injury to the brainstem and cranial neuropathies. In patients with benign tumors, the goal of the operation should be decompression of the brainstem and preservation of cranial nerve function. The link to the video can be found at: https://youtu.be/nk8-VztB0OI.
\end{abstract}

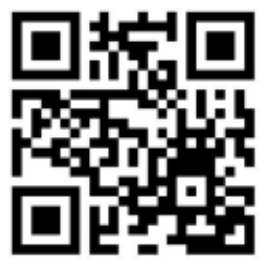

received

June 1, 2018

accepted

August 11, 2018

published online

September 25, 2018

www.thieme.com/skullbasevideos

www.thieme.com/jnlsbvideos

DOI https://doi.org/

10.1055/s-0038-1669980. ISSN 2193-6331.
๑) 2018 Georg Thieme Verlag KG
Stuttgart · New York

License terms

(c) $(1) \ominus$ (\$) 
Disclosure Statement

The authors have no personal financial or institutional interest in any of the materials or devices described in this article.

Conflict of Interest

None.
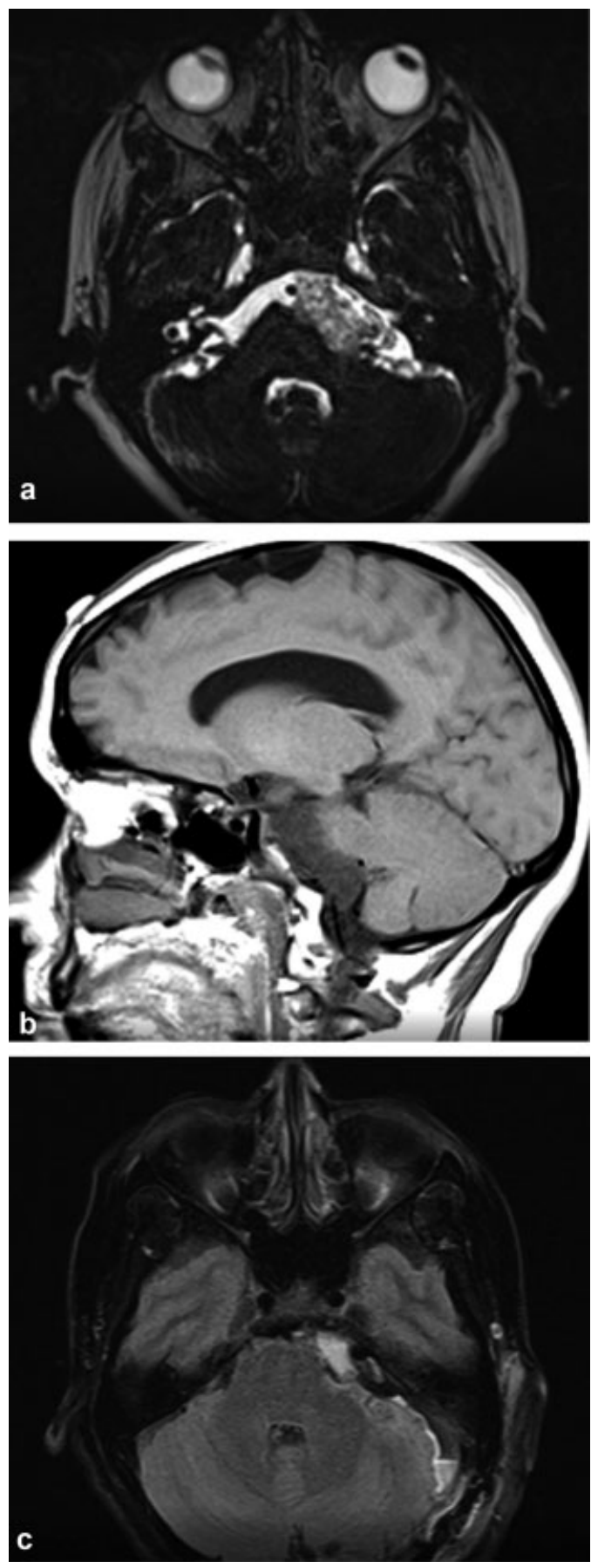

Fig. 1 (a) Axial diffusion-weighted and (b) sagittal T1-weighted magnetic resonance imaging demonstrating a cerebellopontine angle epidermoid cyst with mass effect upon the brainstem. (c) Postoperative axial diffusionweighted magnetic resonance imaging demonstrates complete removal of the lesion.
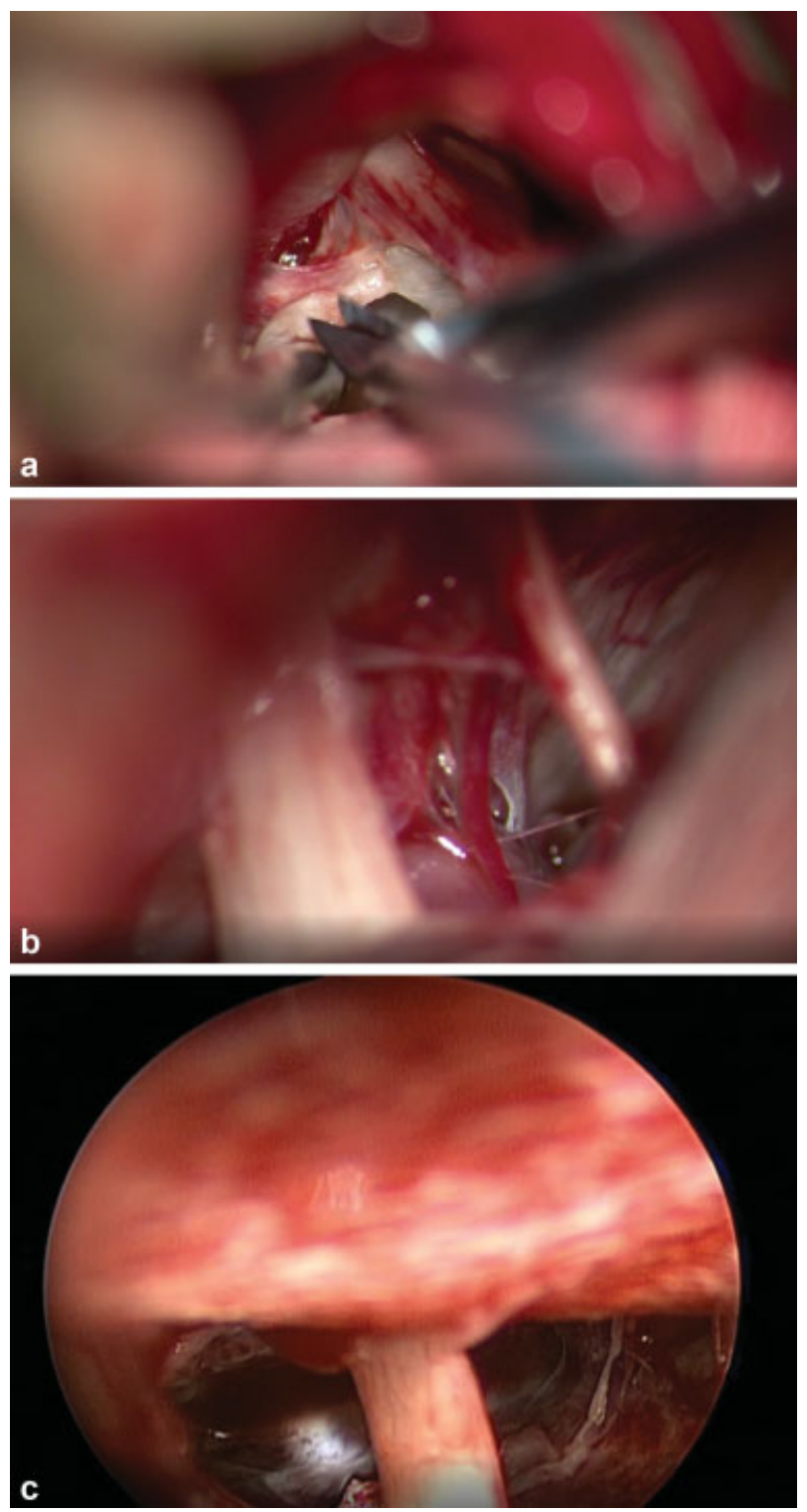

Fig. 2 Intraoperative photographs demonstrating (a) opening of the tumor capsule; (b) visualization of the basilar artery after partial removal of tumor; and (c) endoscopic views of the posterior fossa after resection of the tumor. 